\title{
Positive impacts of e-aid cognitive behavioural therapy on the sleep quality and mood of nurses on site during the COVID-19 pandemic
}

\author{
Kaili Zhou $^{1} \cdot$ Jing Kong ${ }^{2} \cdot$ Yahui Wan $^{1} \cdot$ Xuan Zhang ${ }^{1} \cdot$ Xuan Liu $^{1} \cdot$ Bingqian Qu ${ }^{1} \cdot$ Baoyan Wang ${ }^{1} \cdot$ Rong Xue $^{1,3}$
}

Received: 30 August 2021 / Revised: 26 November 2021 / Accepted: 1 December 2021 / Published online: 8 January 2022

(c) The Author(s), under exclusive licence to Springer Nature Switzerland AG 2022

\begin{abstract}
Objective To investigate the positive impact of e-aid cognitive behavioural therapy on the sleep quality, anxiety, and depression of nurses on site during the COVID-19 pandemic.

Methods Nurses on site at the Tianjin Medical University General Hospital Airport Site experiencing insomnia, anxiety and depression during the COVID-19 prevention and control period, from February 2020 to April 2021, were selected and divided into either an e-aid cognitive behavioural therapy (eCBT-I) group or a control group using a randomized grouping method. The eCBT-I group was given standard eCBT-I for 6 weeks; the control group did not get any intervention. The Pittsburgh Sleep Quality Index (PSQI) and the Insomnia Severity Index (ISI) were used to evaluate the sleep quality of the subjects. The Generalized Anxiety Disorder 7-item (GAD-7) and the Patient Health Questionnaire (PHQ-9) were used to assess the subjects' anxiety and depression. Changes in sleep quality, anxiety and depression before and after treatment were compared between the two groups.

Results Of 118 nurses randomized, the PSQI and ISI scores within the eCBT-I group $(n=60)$ were significantly lower after treatment $(5.9 \pm 3.9,6.7 \pm 4.5)$ than before treatment $(10.4 \pm 3.5,12.4 \pm 4.7)(p<0.05)$. Compared to the scores of the control group $(n=58)(9.1 \pm 3.9,10.6 \pm 4.1)$, the PSQI and ISI scores in the eCBT-I group $(5.9 \pm 3.9,6.7 \pm 4.5)$ were lower after treatment $(p<0.05)$. The GAD-7 and PHQ-9 scores in the eCBT-I group were all lower after treatment $(3.7 \pm 3.4,4.2 \pm 4.1)$ than before treatment $(6.7 \pm 4.9,7.7 \pm 5.1)(p<0.05)$. Compared with subjects in the control group $(7.1 \pm 5.6,7.3 \pm 5.1)$, subjects in the eCBT-I group (3.7 $\pm 3.4,4.2 \pm 4.1)$ had lower scores on the GAD-7 and PHQ-9 scales after treatment $(p<0.05)$. Conclusion eCBT-I improved the sleep quality of frontline nurses during the COVID-19 prevention and control period and relieved anxiety and depression.
\end{abstract}

Keywords E-aid cognitive behavioural therapy $\cdot$ COVID-19 pandemic $\cdot$ Nurses on site $\cdot$ Sleep quality $\cdot$ Anxiety and depression

\section{Introduction}

Rong Xue

xuerong1403@126.com

Kaili Zhou

18249519476@163.com

Jing Kong

kongjing0604@163.com

1 Department of Neurology, Tianjin Medical University General Hospital Airport Site, Tianjin 300000, China

2 Department of Geriatric Medicine, Tianjin Medical University General Hospital Airport Site, Tianjin 300000, China

3 Department of Neurology, Tianjin Medical University General Hospital, Tianjin 300052, China
Since December 2019, COVID-19, which emerged in Wuhan, Hubei Province, China, has spread throughout the country. The COVID-19 pandemic has precipitated life crises for many people and caused psychological stress for many others, including tension, anxiety, fear and despair. In particular, nurses on site have been more prone to these psychological disorders [1]. The lack of experience with infection prevention and control, the high-intensity work, the mental stress, the shift work system, the loneliness and loss caused by isolation, and the lack of psychological counselling have led to a series of physiological and psychological stress reactions, which have seriously affected the sleep and mood of nurses and manifested mainly as insomnia, anxiety 
and depression [2]. Cognitive behavioural therapy for insomnia is a set of treatment strategies that target the behaviour, cognition, and physiology of patients with chronic insomnia. Through changes in patient behaviour, sleep hygiene habits, sleep cognition, and other factors, insomnia can be improved. Cognitive behavioural therapy (CBT-I) has been recommended as a first-line treatment for adult chronic insomnia and insomnia with anxiety and depression [3]. However, traditional group or individual CBT-I is performed by trained doctors or psychotherapists. It has a set of standardized procedures, complex steps and a long treatment duration and thus is difficult to complete during public health emergencies especially when faced with a lack of medical resources. Therefore, it is very important to find a simple and effective psychotherapy. E-aid cognitive behavioural therapy (eCBT-I) has been shown to be a safe, highly effective, and widely available treatment method [4]. However, eCBT-I has not yet reached its full potential in terms of scope and scale. This study aimed to observe the effects of eCBT-I on the sleep quality, anxiety, and depression of nurses on site with chronic insomnia and to provide more clinical evidence for the application of eCBT-I in patients with different degrees of insomnia.

\section{Patients and methods}

\section{Subjects}

Nurses on site with chronic insomnia who agreed to participate in this study were selected from the Tianjin Medical University General Hospital Airport Site during the COVID-19 pandemic prevention period, from February 2020 to April 2021. Inclusion criteria included: (a) male and female nurses who were on site and who participated in COVID-19 pandemic prevention and control; (b) those who met the diagnostic criteria of chronic insomnia in International Classification of Sleep Disorders (ICSD-3)[5]; (c) those between ages 24 and 44 years; (d) those whose use sedative and hypnotic drugs was stopped at the beginning of the treatment period (or gradually discontinued 2 weeks before the study); (e) those who reported no recent major stress events; and (f) those who could understand and follow the research protocol, use the internet, voluntarily participate in the clinical study and sign the informed consent. Exclusion criteria included: (a) those with the presence of a diagnosed somatic disease that may also have caused insomnia; (b) those with severe anxiety, depression, or other mental disorders, who could not cooperate with treatment; (c) those with drug or alcohol abuse or dependence; (d) those who previously received CBT-I; and (e) those who were pregnant or lactating. The above nurses on site were divided by a random grouping method (by lot) into either the eCBT-I group or a control group. The eCBT-I group was given standard eCBT-I for 6 weeks; the control group did not get any intervention. CBT-I courses can only be sent through the platform and cannot be forwarded by patient portal site (via mobile phone or tablet), and the nurses randomized to the intervention group were told not to mention or watch the CBT-I information and techniques with the control group. All procedures performed in studies involving human participants were in accordance with the ethical standards of the institutional and/or national research committee and with the 1964 Helsinki declaration and its later amendments or comparable ethical standards (Medical Ethics Committee of Tianjin Medical University General Hospital). Informed consent was obtained from all participants and their legal guardian.

\section{eCBT-I treatment}

The SUMIAN CBT-I management system was adopted, and CBT-I-trained and experienced medical staff conducted subject label management, customized treatment plans, checked subject implementation and configured follow-up plans according to the subjects' compliance and improvement; batch medical orders were sent, and subjects kept sleep diaries, took CBT-I courses, reviewed sleep reports, used various sleep improvement tools such as relaxation training and communicated with health care providers online through a patient portal site (via mobile phone or tablet). The standard treatment course was 6 weeks, with structured CBT-I animation courses every day. The specific program content included: Week 1--understanding sleep, understanding the causes of insomnia through learning and skilfully mastering the use of sleep recording tools. Week 2-- learning sleep restriction methods, gradually solidifying sleep and striving to make sleep time more sustainable. Week 3--enhancing the association between bed and sleep and shortening the duration of falling asleep through stimulus control exercises. Week 4--identifying insomnia and managing bad moods before sleep through cognitive therapy training. Week 5--learning how to control anxiety and minimize worries before bed to achieve deep sleep. Week 6--preventing relapse and learning to cope with acute insomnia. Before treatment, a professional therapist conducted a remote telephone interview with each subject, and after the interview, a treatment plan was sent to each participant through the platform at least once per week for communication and interaction, thus enabling a dynamic understanding of the treatment status of each subject.

\section{Assessment methods}

Assessments of sleep quality, anxiety, and depression were performed by a trained doctor in an evaluator-blinded 
fashion. The two groups of subjects were evaluated before and after treatment. Sleep assessment included the following: (a) sleep quality was assessed using the Pittsburgh Sleep Quality Index (PSQI), a self-rated questionnaire that is scored on a scale of 0 to 21 , with a score $>5$ indicating impaired sleep quality, and with higher scores indicating greater impairment of sleep [6,7]; (b) the severity of insomnia was assessed using the Insomnia Severity Index (ISI) [8], with total scores ranging from 0 to 28: no clinically significant insomnia ( $0-7$ points); subthreshold insomnia (8-14 points); moderate insomnia (15-21 points); severe insomnia (22-28 points). Assessment of anxiety and depression included: (a) the severity of anxiety was assessed using the Generalized Anxiety Disorder 7-item (GAD-7), which is a brief seven-item screening questionnaire that has been found to be sensitive to generalized anxiety disorder, social phobia and panic disorder, with higher scores indicating greater symptom severity. The internal consistency of the GAD-7 scale is good. The GAD-7 has good convergent and divergent validity with other anxiety and disability scales. A clinical cutoff score of 8 indicates a diagnosis of anxiety disorder [9]. (b) The severity of depressive symptoms was assessed using the Patient Health Questionnaire-9 (PHQ-9), which has an overall score ranging from 0 to 27 , with $0-4$ indicating not depressed, 5-9 indicating mildly depressed, 10-14 indicating moderately depressed, 15 to 19 indicating moderate to severe depression and 20 to 27 indicating severe depression [10].

\section{Sample size estimation}

In most CBTI studies, sleep efficiency by sleep diary is greater than $85 \%$ as the clinical cure rate index, while in a few studies, ISI is less than 8 as the clinical cure rate index [11-13]; the proportion of patients with sleep efficiency greater than $85 \%$ after treatment is about $60 \%$, and the proportion of patients with ISI less than 8 is about $40 \%$. In this study, a strict standard ISI less than 8 was used to estimate the sample size by statistical and confidence analysis software (PASS), where $\alpha=0.05, \beta=0.1$, mean difference $=2.0$ and standard deviation difference $=3.0$. The sample size of each group was determined to be at least 45 .

\section{Statistical analysis}

SPSS statistical software was used to process and analyse the data, and results were expressed as the mean \pm standard deviation (SD) for continuous variables. $T$-tests were used before and after treatment; differences in demographics, past medical history and sleep characteristics between the two groups were compared descriptively using chi-square tests for categorical measures. A value of $p<0.05$ indicated that the difference was statistically significant.

\section{Results}

Of 118 nurses randomized, there were 60 nurses (59 women) in the eCBT-I group, with an average age of 31.0 \pm 4.4 years; there were 58 nurses ( 57 women) in the control group, with an average age of $29.6 \pm 4.5$ years. Between the two groups, there were no significant differences in age, sex, height, weight, education, smoking history, drinking history, history of hypertension, diabetes, coronary heart disease, sleep disorders, family history of mental illness, course of disease, time spent fighting the pandemic or working hours, indicating that the two groups were comparable.

\section{Sleep scale scores before and after treatment}

PSQI and ISI scores in the eCBT-I group were significantly lower after treatment than those before treatment $(p<0.05)$. Compared with the control group, the PSQI and ISI scores in the eCBT-I group were lower after treatment $(p<0.05)$. The details of the sleep characteristics are summarized in Table 1.

\section{Anxiety and depression scale scores before and after treatment}

The GAD-7 and PHQ-9 scores in the eCBT-I group were lower after treatment than those before treatment $(p<0.05)$. Compared with those of the control group, the scores of the Broad Anxiety Scale and PHQ-9 scales in the eCBT-I group were decreased after treatment $(p<0.05)$ (Table 2).

\section{Discussion}

After 6 weeks of treatment, the sleep quality, anxiety, and depression of frontline nurses during COVID-19 prevention and control were significantly improved, suggesting that eCBTI had good clinical efficacy.

Table 1 Sleep scale scores before and after treatment

\begin{tabular}{llllll}
\hline & \multicolumn{2}{l}{ eCBT-I group $(n=60)$} & & \multicolumn{2}{l}{ Control group $(n=58)$} \\
\cline { 2 - 3 } \cline { 6 - 7 } & Baseline & Study end & & Baseline & Study end \\
\hline PSQI & $10.4 \pm 3.5$ & $5.9 \pm 3.9^{*, * *}$ & & $10.0 \pm 3.1$ & $9.1 \pm 3.9$ \\
ISI & $12.4 \pm 4.7$ & $6.7 \pm 4.5^{* * * *}$ & & $11.8 \pm 3.4$ & $10.6 \pm 4.1$ \\
\hline
\end{tabular}

Data shown are the mean $\pm \mathrm{SD}$

Abbreviations: $e C B T-I$ e-aid cognitive behavioural therapy; $P S Q I$ Pittsburgh Sleep Quality Index; ISI Insomnia Severity Index

${ }^{*} p<0.05$ : comparison of between the study end and baseline values of the eCBT-I group; ${ }^{* *} p<0.05$ : comparison of study end values between the eCBT-I group and control group 
Table 2 Anxiety and depression scale scores before and after treatment

\begin{tabular}{llllll}
\hline & \multicolumn{2}{l}{ eCBT-I group $(n=60)$} & & \multicolumn{2}{l}{ Control group $(n=58)$} \\
\cline { 2 - 3 } \cline { 6 - 6 } & Baseline & Study end & & Baseline & Study end \\
\hline GAD-7 & $6.7 \pm 4.9$ & $3.7 \pm 3.4^{*, * *}$ & & $7.6 \pm 5.1$ & $7.1 \pm 5.6$ \\
PHQ-9 & $7.7 \pm 5.1$ & $4.2 \pm 4.1^{*, * *}$ & & $8.2 \pm 4.4$ & $7.3 \pm 5.1$ \\
\hline
\end{tabular}

Data shown are the mean \pm SD

Abbreviations: $e C B T-I$ e-aid cognitive behavioural therapy; $G A D-7$ Generalized Anxiety Disorder 7-item; PHQ-9 Patient Health Questionnaire-9

${ }^{*} p<0.05$ : comparison of between the study end and baseline values of the eCBT-I group; ${ }^{* *} p<0.05$ : comparison of study end values between the eCBT-I group and control group

A large number of studies have confirmed that eCBT-I is effective in the treatment of insomnia and can effectively increase sleep efficiency and improve sleep quality $[14,15]$. Consistent with previous research results, our study showed that after treatment, the PSQI and ISI scores of subjects in the eCBT-I group after treatment were significantly lower than those before treatment and significantly different from those in the control group, indicating that eCBT-I improved the sleep quality of nurses on site with chronic insomnia during the prevention and control period of COVID-19.

eCBT-I has been shown to reduce anxiety and depression symptoms [16]. A study of the effect of symptoms of depression and anxiety on insomnia improvements in 455 patients during CBT-I showed that symptoms of depression and anxiety did not impair the effectiveness of CBT-I. Instead, CBT-I was associated with moderate-to-large improvement of depression and anxiety symptoms in patients with insomnia disorder [17]. Findings of the current study were consistent with previous research results. These results indicate that eCBT-I can relieve anxiety and depression in nurses with chronic insomnia on the front line of COVID-19 prevention and control. The focus of treatment is on helping patients change their adverse cognitive patterns. On the one hand, it may be the effect of the treatment itself; imaging studies suggest that the early stage of CBT may modulate functional connectivity between subgenual anterior cingulate cortex and inferior frontal gyrus and some nodes of default mode network, which result in enhanced response inhibition and reduced self-referential process and rumination of depressed patients [18]. On the other hand, it may be the additional effect of improved sleep, sleep disorder, anxiety and depression affect each other, and the improvement of sleep gradually causes the change of mood regulation.

Although the present study provides some valuable findings, its design imposes the limitations: (1) considering convenience, the control group in the study was blank control rather than placebo control, so it is difficult to exclude a placebo effect caused by the "Hawthorne effect". (2) The participants were almost exclusively women. The results of this study are therefore not fully generalizable to the population at large or other settings that include more men. The male sample size will be increased for further study in the future. In addition, the long-term effect of this method in this population needs further observation.

\section{Conclusion}

Thus, during the COVID-19 prevention and control period, eCBT-I was widely applied to front-line medical workers to improve their sleep and mood, ensure their physical and mental health, and promote their better participation in the prevention and control of the pandemic. At the same time, this study also provided a basis for the application of eCBT-I among different populations of insomnia patients, which is worthy of further promotion.

Author contribution Guarantor of integrity of the entire study: Kaili Zhou, Jing Kong and Rong Xue. Study concept: Kaili Zhou, Jing Kong and Rong Xue. Study design: Kaili Zhou, Jing Kong and Rong Xue. Literature research: Xuan Zhang, Yahui Wan, Xuan Liu and Baoyan Wang. Clinical assessment: Kaili Zhou and Jing Kong. eCBT-I examination: Kaili Zhou and Bingqian Qu. Data analysis: Kaili Zhou. Manuscript preparation: Kaili Zhou. Manuscript review: Rong Xue.

Funding This study was funded by the Major project of Tianjin science and technology, prevention and control technology of chronic disease (17ZXMFSY00180).

Data availability The data that support the findings of this study are available from the corresponding author upon reasonable request.

\section{Declarations}

Ethics approval All procedures performed in studies involving human participants were in accordance with the ethical standards of the institutional and/or national research committee and with the 1964 Helsinki declaration and its later amendments or comparable ethical standards (Medical Ethics Committee of Tianjin Medical University General Hospital).

Informed consent Informed consent was obtained from all individual participants included in the study.

Conflict of interest The authors declare no competing interests.

\section{References}

1. Song M (2020) Psychological stress responses to COVID-19 and adaptive strategies in China - ScienceDirect. World Development 136:105-107. https://doi.org/10.1016/j.worlddev.2020.105107

2. Maiorano T, Vagni M, Giostra V, Pajardi D (2020) COVID-19: risk factors and protective role of resilience and coping strategies for emergency stress and secondary trauma in medical staff 
and emergency workers - an online-based inquiry. Sustainability 12(21):1-18

3. Qaseem A, Kansagara D, Forciea MA, Cooke M, Denberg TD (2016) Management of chronic insomnia disorder in adults: a clinical practice guideline from the American College of Physicians. Ann Intern Med 165(2):125-133. https://doi.org/10.7326/ M15-2175

4. van der Zweerde T, Lancee J, Ida LA, van Straten A (2020) Internet-delivered cognitive behavioral therapy for insomnia: tailoring cognitive behavioral therapy for insomnia for patients with chronic insomnia. Sleep Med Clin 15(2):117-131. https://doi.org/ 10.1016/j.jsmc.2019.04.002

5. Sateia MJ (2014) International classification of sleep disordersthird edition: highlights and modifications. Chest 146(5):13871394. https://doi.org/10.1378/chest.14-0970

6. Magro R, Camilleri L, Borg A (2020) Fri0178 predictive factors for poor sleep quality in systemic lupus erythematosus. Ann Rheumatic Dis 79(1):673.2-673. https://doi.org/10.1136/ annrheumdis-2020-eular.931

7. Wennberg A, Lorusso R, Dassie F, Benavides-Varela S, Parolin M, De Carlo E, Fallo F, Mioni R, Vettor R, Semenza C, Maffei $\mathrm{P}$ (2019) Sleep disorders and cognitive dysfunction in acromegaly. Endocrine 66(3):634-641. https://doi.org/10.1007/ s12020-019-02061-1

8. Dieperink KB, Elnegaard CM, Winther B, Lohman A, Zerlang I, Moller S, Zangger G (2020) Preliminary validation of the insomnia severity index in Danish outpatients with a medical condition. J Patient Rep Outcomes 4(1):18. https://doi.org/10.1186/ s41687-020-0182-6

9. Johnson SU, Ulvenes PG, Oktedalen T, Hoffart A (2019) Psychometric properties of the General Anxiety Disorder 7-item (GAD7) scale in a heterogeneous psychiatric sample. Front Psychol 10:1713-1713. https://doi.org/10.3389/fpsyg.2019.01713

10. Ganz PA, Bower JE, Partridge AH, Wolff AC, Thorner ED, Joffe H, Irwin MR, Petersen L, Crespi CM (2021) Screening for depression in younger breast cancer survivors: outcomes from use of the Patient Health Questionnaire-9 (PHQ-9). JNCI Cancer Spectrum 5(3):1-8. https://doi.org/10.1093/jncics/pkab017

11. Zachariae R, Lyby MS, Ritterband LM, O'Toole MS (2016) Efficacy of internet-delivered cognitive-behavioral therapy for insomnia - a systematic review and meta-analysis of randomized controlled trials. Sleep Med Rev 30:1-10. https://doi.org/10.1016/j. smrv.2015.10.004
12. Cheng SK, Dizon J (2012) Computerised cognitive behavioural therapy for insomnia: a systematic review and meta-analysis. Psychother Psychosom 81(4):206-216. https://doi.org/10.1159/00033 5379

13. Chen PY, Jan YW, Yang CM (2017) Are the Insomnia Severity Index and Pittsburgh Sleep Quality Index valid outcome measures for cognitive behavioral therapy for Insomnia? Inquiry from the perspective of response shifts and longitudinal measurement invariance in their Chinese versions. Sleep Med 35, 35(1):-40. https://doi.org/10.1016/j.sleep.2017.04.003

14. Lopez R, Evangelista E, Barateau L, Chenini S, Dauvilliers Y (2019) French language online cognitive behavioral therapy for insomnia disorder: a randomized controlled trial. Front Neuro 110:1273. https://doi.org/10.3389/fneur.2019.01273

15. Seyffert M, Lagisetty P, Landgraf J, Chopra V, Pfeiffer PN, Conte ML, Rogers MA (2016) Internet-delivered cognitive behavioral therapy to treat insomnia: a systematic review and meta-analysis. PLoS One 11(2):e0149139. https://doi.org/10.1371/journal.pone. 0149139

16. Ye YY, Zhang YF, Chen J, Liu J, Li XJ, Liu YZ, Lang Y, Lin L, Yang XJ, Jiang XJ (2015) Internet-based cognitive behavioral therapy for insomnia (ICBT-i) improves comorbid anxiety and depression-a meta-analysis of randomized controlled trials. PLoS One 10(11):e142258. https://doi.org/10.1371/journal. pone. 0142258

17. Sweetman A, Lovato N, Micic G, Scott H, Bickley K, Haycock J, Harris J, Gradisar M, Lack L (2020) Do symptoms of depression, anxiety or stress impair the effectiveness of cognitive behavioural therapy for insomnia? A chart-review of 455 patients with chronic insomnia. Sleep Med 75:401-410. https://doi.org/10.1016/j. sleep.2020.08.023

18. Teng CJ (2016) Effect of cognitive behavior therapy on functional connectivity of subgenual anterior cingulated cortex in firstepisode treatment-na6ve mild to moderate patients with major depressive disorder. Chin Med Abstracts (Internal Medicine) 33(4):236-237 CNKI:SUN:ZYNE.0.2016-04-081

Publisher's note Springer Nature remains neutral with regard to jurisdictional claims in published maps and institutional affiliations. 